\title{
PESQUIMAT
}

Revista del Insituto de

Investigación de la Facultad

de Ciencias Matemálicas de la UNMSM

Vol I, N I, pp 103-128 Lima- Perú Julio-1998

\section{'EXPLORAÇÃO DE PROJETOS DE RECURSOS NATURAIS: AVALIAÇĀO E GESTÃO PELA TEORIA DE ATIVOS CONTINGENTES E SIMULAÇÃO}

\author{
Carlos Patricio Samanéz (*) \\ Savio Henrique G. Levi (**)
}

Sumário: 1. Introdução; 2. Incerteza econômica; 3. A dinâmica do crescimento da floresta; 4. O fluxo de caixa gerado pelo corte das árvores; 5. Avaliação por meio da abordagem de ativos contingentes; 5.1. Determinação do valor do projeto; 5.2. Condições de contorno; 5.3. Aplicação à avaliação de uma concessão para a exploração de uma reserva florestal; 5.4. Apresentação e análise dos resultados; 6. Avaliação por meio de simulação - valor presente estratégico; 6.1. Apresentação dos resultados obtidos; 7. Conclusões.

Palavras-chave: análise econômica de projetos; opções reais; controle ótimo estocástico; finanças em tempo contínuo,

Neste artigo os problemas de valoração de projetos de exploração florestal em condições de incerteza são resolvidos no contexto da análise de ativos contingentes, empregando argumentos de inexistência de arbitragem, e através do cálculo do Valor Presente Líquido Estratégico. A Flexibilidade gerencial existente é modelada por uma série de opções relacionadas ao timing do investimento, e à escolha de uma politica de produção ótima. Avaliam-se concessões supondo a existência de incerteza econômica quanto ao preço da madeira, e incerteza técnica quanto à taxa de crescimento da floresta, ambas modeladas através de processos estocásticos em tempo contínuo. Alguns dos modelos matemáticos desenvolvidos não possuem solução analítica conhecida. Resultados são então obtidos através da aproximação numérica das equações diferenciais parciais utilizando fórmulas de diferenças finitas e também através da aproximação numérica direta dos processos estocásticos base utilizando simulação Monte Carlo. Esta metodologia pode ser geralmente utilizada na análise de oportunidades de investimento em condições de incerteza.

In this paper the valuation problems of forestry resources explotation projects under uncertainty are solved under arbitrage free arguments employed in a contingent claims analysis framework, and by calculating the Strategic-NPV. The existing managerial flexibility are modeled by a series of options related to investment timing, and to the choice of an optimal production policy. The leasehold valuation problems takes into account the economic uncertainty in the timber price and the technical uncertainty in the timber growth rate, both modeled through continuous time stochastic processes. Unfortunately, some of the developed mathematical models have no known analytical solutions. Results are then found by numerical aproximation of the Partial Diferential Equations using finite difference formulae, as well as, by direct numerical aproximation

* Professor do Departamento de Engenharia Industrial ja PUC/Rio e da Faculdade de Ciencias Economicas da UERJ.

** Software Erigineer - Fábrica Digital - Projeto Génesis - PUC/Rio 
of the underlying stochastic processes using Monte Carlo simulation. This methodology may be generally applied to value investments opportunities under uncertainty.

\section{1- Introduçăo}

A aplicação da teoria das opçð̃es análise de investimentos vem despertando interesse tanto no meio acadêmico quanto nas empresas, porque consegue explicar certas decisð̄es de investimento e avaliaçð̄es subjetivas feitas no mundo empresarial năo explicáveis pelos métodos tradicionais.

Ao contrário dos métodos tradicionais de desconto de fluxos de caixa, que supõem uma postura passiva da gerência frente aos acontecimentos futuros, e portanto podem falhar na presença de irreversibilidade ou de incerteza, esta metodologia leva em considerą̧ão que a gerência irá atuar no futuro maximizando o lucro da firma, em qualquer estado da natureza que venha a ocorrer durante a vida útil de um projeto.

Através da modelagem da incerteza por processos estocásticos e da modelagem da flexibilidade operacional e financeira por uma série de op̧̧̃̃es à disposição do gerenciamento dos projetos, é possivel levar em consideração na análise econômica a irreversibilidade dos investimentos e parte da flexibilidade e da incerteza ęxistentes no mundo real.

Este artigo trata da análise econômica de projetos de exploração florestal visando a produção de celulose, em condições de incerteza econômica, aplicando a Teoria de Opções Reais ou Análise de Ativos Contingenciados e a Simulação. A metodologia utilizada, embora descrevendo um projeto de exploração florestal, pode ser aplicada para outros casos similares de exploração de recursos naturais.

\section{2- Incerteza Ecònômica ${ }^{3}$}

Existe incerteza econômica a respeito do preço das commodities em geral, e este tipo de incerteza tem correlação com o desempenho da economia como um todo. A incerteza econômica será suposta exógena ao processo decisório e modelada por um processo estocástico estacionário em tempo contínuo do tipo Movimento Geométrico Browniano, 
padrão na literatura de finanças para modelar a incerteza na variação dos preços de uma commodity.

Seja $\mathbf{t}$ a variável que representa o tempo corrente e $\mathbf{P}$ a variável que representa o preço da madeira. Considerando que toda a incerteza sobre oferta e demanda no futuro seja refletida na variação aleatória dos preços da madeira, a sua dinâmica pode ser descrita pela seguinte equação diferencial estocástica:

$$
d \mathbf{P} / \mathbf{P}=\mu_{\mathrm{p}} \mathbf{d t}+\sigma_{\mathrm{p}} \mathbf{d} \mathbf{z}_{\mathbf{p}}
$$

$\mathbf{P}$ representa o preço da madeira;

t representa o tempo;

$\mu_{p}$ é o coeficiente de "drift ";

$\sigma_{p}$ é o coeficiente de volatilidade ou desvio padrão;

$\mathrm{dz}_{\mathrm{p}}$ é um processo de Itô, $\mathrm{dz} \mathrm{p}_{\mathrm{p}}=\varepsilon \sqrt{ }(\mathrm{dt}) ;$

$\varepsilon \sim N(0,1)$ é uma variável aleatória com distribuição normal, média zero e variância 1 . Os parâmetros $\mu_{p}$ e $\sigma_{p}$, necessários para empregar a equação (1), podem ser estimados com base em séries históricas de preços.

\section{3- A dinâmica do crescimento e corte da floresta}

Seja $\mathbf{E}$ a variável que representa o estoque de madeira, sendo que a reserva florestal possui inicialmente $\mathbf{E}_{O}$ metros cúbicos de madeira. Seja $\mathrm{Q}(\mathrm{P}, \mathrm{E}, \mathrm{t})$ a taxa instantânea de corte de madeira, sendo que $Q(P, E, t)$ pode ser uma função qualquer do preço, do estoque e do tempo.

A dinâmica do estoque pode ser descrita pela seguinte equação diferencial estocástica:

$$
d E=\left[\mu_{e}(E, t)-Q(P, E, t)\right] d t+\sigma_{e}(E, t) d z_{e}
$$

Ną equação acima:

dE é a variação infinitesimal do estoque;

t representa o tempo; 
$\left[\mu_{c}(E, t)-Q(P, E, t)\right]$ é o coeficiente de "drift ";

$\sigma_{\mathrm{e}}(\mathrm{E}, \mathrm{t})$ é o coeficiente de volatilidade ou desvio padrão;

$\mathbf{d z}_{\mathrm{e}}$ é um processo de Wiener, $d z_{\mathrm{e}}=\varepsilon \sqrt{ }(\mathrm{dt})$;

$\varepsilon \sim \mathrm{N}(0,1)$ é una variável aleatória com distribuição normal. média zero e variância 1 .

De acordo com a equação (2), o estoque cresce a uma taxa instantânea aleatória que possui média $\mu_{\mathrm{c}}(\mathrm{E}, \mathrm{t})$ e desvio padrão $\sigma_{\mathrm{c}}(\mathrm{E}, \mathrm{t})$ devido ao crescimento das árvores, e simultaneamente decresce a uma taxa instantânea $\mathrm{Q}(\mathrm{P}, \mathrm{E}, \mathrm{t})$ devido ao corte das árvores.

Na realidade, pode-se esperar relações complexas entre $\mu_{e}, \sigma_{e}$ e E. Neste trabalho, para simplificar, $\mu_{\mathrm{c}}(\mathrm{E}, \mathrm{t})$ será suposto igual a $\mu \mathrm{E}$; onde $\mu$ é constante $e, \sigma_{\mathrm{c}}(\mathrm{E}, \mathrm{t})$ será suposto igual a $\sigma \mathrm{E}$; onde $\sigma$ é também constante. Os parâmetros necessários para empregar a equação (2), a taxa de crescimento médio, $\mu_{\mathrm{e}}(\mathrm{E}, \mathrm{t})$, e o seu desvio padrão, $\sigma_{\mathrm{e}}(\mathrm{E}, \mathrm{t})$, são geralmente conhecidos dos Engenheiros Florestais.

É suposto também que os processos estocásticos (1) e (2) não tem correlação, ou seja $\mathbf{d z}_{\mathbf{c}} \mathbf{d z _ { p }}=0$. Deste modo, a variação do estoque de árvores da floresta considerada não influencia o preço de mercado da madeira. Esta hipótese também chamada hipótese da firma pequena, ou " small firm ", significa considerar o preço de mercado como um dado externo ou exógeno ao problema.

\section{4- $O$ fluxo de caixa gerado pelo corte das árvores}

Para descrever o fluxo de caixa gerado pelo corte e venda das toras, é preciso antes definir os custos operacionais e a incidência de impostos. No caso geral, os custos operacionais dependem da taxa de corte $\mathbf{Q}(\mathrm{P}, \mathrm{E}, \mathrm{t})$ e do tempo. Neste trabalho é suposto que os custos operacionais, denominados $\pi(t, \mathbf{Q})$, são conhecidos e representados do seguinte modo:

$\pi(t, Q)=c_{0}(t)+c_{1}(t, Q)$, sendo $c_{0}$ o coeficiente de custo fixo e $c_{1}$ o coeficiente de custo variável. 
O fluxo de caixa gerado pelo corte e posterior venda das toras, antes da incidência dos impostos, pode ser descrito como:

$$
\mathbf{f}=[\mathbf{P} \times \mathbf{Q}(\mathrm{P}, \mathrm{E}, \mathrm{t})] \cdot \pi(\mathrm{t}, \mathrm{Q})
$$

\section{1-Impostos}

Os impostos na realidade incidem sobre o fluxo de caixa das empresas em tempo discreto e são portanto difíceis de modelar em tempo contínuo. Um tratamento realista da legislação vigente provavelmente exigiria a introdução de outra variável de estado dependente do tempo, o que tornaria mais complexo a modelagem e a sua posterior solução numérica. Para simplificar, será considerado apenas a incidência de uma aliquota única de imposto, $\gamma$, sobre o faturamento bruto.

O fluxo de caixa instantâneo após a incidência dos impostos, $F(P, Q, V, \pi ; \gamma$ ), será:

$$
\mathbf{F}=\{(1-\mathrm{tc})[(1-\mathrm{tr}) \times \mathbf{P} \times \mathbf{Q}(\mathrm{P}, \mathrm{E}, \mathrm{t})]-\pi(\mathrm{t}, \mathrm{Q})\}-\lambda \mathbf{V} \quad \text { se } Q(P, E, t)>0
$$

Admitimos que existe a possibilidade de se exercer a opção de suspender temporariamente a produção, ou seja pode ocorrer que $Q(P, E, t)=0$,

\section{5- Avaliação por meio da abordagem de ativos contingentes}

Em finanças, uma opção financeira é chamada ativo derivativo ou contingente, porque o seu preço depende do preço de outro ativo, no caso as ações da empresa, chamado ativo primário. No caso do projeto considerado neste trabalho, supõe-se que a madeira é uma commodity negociada nos mercados à vista e futuro, com liquidez. $\mathrm{O}$ preço da madeira é neste caso o ativo primário, e o valor da concessão ou direito de exploração é o ativo derivado ou contingente.

-Consideremos um portfolio denominado $\phi$, composto de uma posição longa em uma unidade do direito de explorar a reserva florestal (ativo derivativo ), e de uma posição 
curta em $n$ unidades da commodity (ativo primário). Se V é o valor unitário do ativo derivativo e P o preço unitário do ativo primário, o valor do portfolio será o seguinte:

$$
\phi=1 \times V(P, E, t)-n \times P
$$

Ao manter o portfolio $\phi$ durante um pequeno intervalo de tempo, $(t, t+d t)$, a posição longa retorna ao investidor um fluxo de dividendos, Fdt, ou seja, o fluxo de caixa liquido instantâneo recebido por cortar e vender as árvores. Para manter a posição curta, é preciso que se desembolse $(n \times \mathbf{P} \times \delta$ )dt, sendo $\delta$ um custo de oportunidade, caso contrário nenhum investidor racional entraria na outra ponta do negócio. Deste modo, o dividendo liquido recebido (fluxo de caixa) por se manter o portfolio $\phi$ por este pequeno intervalo de tempo, $(\mathrm{t}, \mathrm{t}+\mathrm{dt})$, é igual a $(\mathbf{F}-n \times \delta \times \mathbf{P}) \mathrm{dt}$.

A madeira é uma commodity estocável, logo o custo de oportunidade ò deve ser igual ao retorno de conveniência ( convenience yield) liquido resultante da sua estocagem ${ }^{4}$, denominado $\kappa$.

Ao manter-se o portfolio $\phi$ por este pequeno intervalo de tempo, $(t, t+d t)$, recebese, além dos dividendos descritos acima, um ganho de capital (valorização do projeto) representado da seguinte forma:

$$
\mathrm{d} \phi=\mathrm{dV}-n \times \mathrm{dP}
$$

O retorno total deste portfolio, $\mathrm{R}_{\mathrm{p}}$, é portanto a soma das parcelas correspondentes ao ganho de capital e aos dividendos:

$$
\mathrm{R}_{\mathrm{p}}=(\mathrm{dV}-n \times \mathrm{dP})+\left(\mathrm{F}-n \times \delta_{\mathrm{p}} \times \mathrm{P}\right) \mathrm{dt}
$$

Aplicando-se o lema de Itô para calcular dV $(P, E, t):^{5}$

\footnotetext{
4 o convenience yield líquido resultante da estocagem de uma commodity é definido como sendo o fluxo de beneficios, menos os custos de estocagem, que a unidade marginal estocada produz. Estes beneficios podem incluir a abilidade de evitar a falta temporária de matérias primas, tirar proveito de flutuaçőes nos preços, evitar flutuaçōes na produçào assim como facilitar a programação da produção e as vendas. Para a maioria das commodities, 0 convenience yield marginal varia inversamente com a quantidade total estocada. Para um estudo mais detalhado, veja Brennan [4].

${ }^{5} \mathrm{O}$ lema permite expressar uma variável aleatória em função de outra. $V_{1}, V_{e}, V_{p}, V_{e e} e V_{p p}$ representam derivadas parçiais.
} 


$$
d V=V_{t} d t+V_{p} d P+1 / 2 V_{p p}(d P)^{2}+V_{e} d E+1 / 2 V_{e c}(d E)^{2}
$$

Substituindo (2) e (8) em (7) e considerando $\delta_{p}=\kappa$ :

$$
\mathrm{R}_{\mathrm{p}}=\left(\mathrm{F}-n \mathrm{kP}+\mathrm{V}_{1}+1 / 2 \sigma_{\mathrm{p}}^{2} \mathrm{P}^{2} \mathrm{~V}_{\mathrm{pp}}+1 / 2 \sigma_{\mathrm{e}}^{2} \mathrm{E}^{2} \mathrm{~V}_{\mathrm{ee}}+[\mu \mathrm{E}-\mathrm{Q}] \mathrm{V}_{\mathrm{e}}\right) \mathrm{dt}+\left(\mathrm{V}_{\mathrm{p}}-n\right)+
$$

$\mathrm{dP} \underline{\underline{E} \mathrm{EV}_{e}} \underline{\mathrm{dze}}$

$\mathrm{O}$ investidor não espera retorno proveniente da parcela sublinhada na equação acima, $\mu \mathrm{Ev}_{\underline{c}} \underline{\mathrm{dze}}$, porque esta parcela representa a incerteza técnica sobre a taxa de crescimento das árvores, este termo na verdade não contribui para o retorno total do portfolio. Argumento similar é utilizado por Dixit e Pindyck [6], ${ }^{6}$ num projeto que também apresenta incerteza técnica e econômica simultaneamente, para ignorar a parcela do retorno total do portfolio que depende apenas da incerteza técnica. ${ }^{7}$

Faz-se então: $n=V_{p}$, deste modo a composição do portfolio é mantida constante durante o pequeno intervalo de tempo $(t, t+d t)$ de modo que $n$ permaneça igual a $\mathrm{V}_{\mathrm{p}}(\mathrm{t})$ apesar de $V_{p}$ variar durante este intervalo. Com o passar do tempo, esta estratégia de hedge dinâmico irá ajustar o portfolio de modo que fique sempre livre de risco.

Dixit e Pindyck [6], indicam os trabalhos de Harrison e Kreps [1979] e Duffie [1988] para a formulação matemática rigorosa desta estratégia de Hedge dinâmica no limite quando $\mathrm{dt} \rightarrow 0$. Se o portfolio $\phi$ é livre de risco durante um intervalo de tempo dt, então seu retorno deve ser igual a $\mathbf{r} \phi$ dt para não existirem oportunidades de arbitragem, onde $r$ é a taxa de juros sem risco.

Simplificando e substituindo o valor do fluxo de caixa $\mathbf{F}$ segundo a equação (4), obstem-se:

$$
\begin{aligned}
& \left(1-\tau_{c}\right)\left[\left(1-\tau_{r}\right) P Q-c_{0}-c_{1} Q\right]-(\lambda+r) V+V_{t}+(r-k) P V_{p}+[\mu E-Q] V_{c}+1 / 2 \sigma_{p}{ }^{2} P^{2} V_{p p}++ \\
& 1 / 2 \sigma_{e}^{2} E^{2} V_{e e}=0 \\
& 7
\end{aligned}
$$




\section{1- Determinação do valor do projeto}

O valor do projeto será determinado no contexto de um problema de controle ótimo estocástico para a taxa de corte de madeira. Deste modo, será igual ao valor da opção de cortar as árvores no melhor momento possível. Pode-se escrever então a seguinte equação de Bellman no contexto de um problema de controle ótimo em relação a taxa de corte $\mathrm{Q}(\mathrm{P}, \mathrm{E}, \mathrm{t})$ :

\section{$\operatorname{Max}$}

$Q \in[0$, maior taxa de produção tecnicamente possivel $(\beta)]$

$$
\begin{aligned}
& \left\{\left(1-\tau_{c}\right)\left[\left(1-\tau_{\mathrm{r}}\right) \mathrm{PQ}-\mathrm{c}_{0}-\mathrm{c}_{1} \mathrm{Q}\right]-(\lambda+\mathrm{r}) \mathrm{V}+\mathrm{V}_{\mathrm{t}}+(\mathrm{r}-\kappa) \mathrm{PV} \mathrm{p}+[\mu \mathrm{E}-\mathrm{Q}] \mathrm{V}_{\mathrm{e}}+1 / 2 \sigma_{\mathrm{p}}{ }^{2} \mathrm{P}^{2} \mathrm{~V}_{\mathrm{pp}}+\right. \\
& \left.1 / 2 \sigma_{\mathrm{c}}{ }^{2} \mathrm{E}^{2} \mathrm{~V}_{\mathrm{ce}}\right\}=0
\end{aligned}
$$

A Equação (11) é linear em Q, isto implica que a taxa de corte ótima, a taxa de corte que maximiza o valor do projeto, denominada $\mathrm{q}^{*}$, será do tipo "Tudo ou Nada "8 ${ }^{8}$. Caso similar é discutido por Dixit e Pindyck [6] pp. 348-349. O valor de $q^{*}$ pode assumir os valores:

$$
\begin{array}{ll}
\mathrm{q}^{*}=\beta \text { para } & \left(1-\tau_{\mathrm{c}}\right)\left[\left(1-\tau_{\mathrm{r}}\right) \mathrm{P}-\mathrm{c}_{\mathrm{l}}\right]-\mathrm{V}_{\mathrm{e}} \geq 0 \\
\mathrm{q}^{\bullet}=0 & \text { caso contrário }
\end{array}
$$

Existe uma superfície $\xi$ que divide o espaço \{ preço, estoque, tempo \} em dois subespaços, como pode ser observado nas figuras 2 e 3 . Num destes subespaços vale a equação (12) e portanto $q^{*}=\beta$, no outro subespaço vale a equação (13), e portanto $q^{*}=0$; entretanto, existe a opção de retomar a produção enquanto não acabar a concessão.

\footnotetext{
${ }^{8}$ A equaçào (11) é linear em $Q$ porque a funçăo de custo $\pi(Q)$ é linear em $Q$, caso contrário a taxa de corte ótima $q^{*}$ năo será do tipo "Tudo ou Nada ".
} 


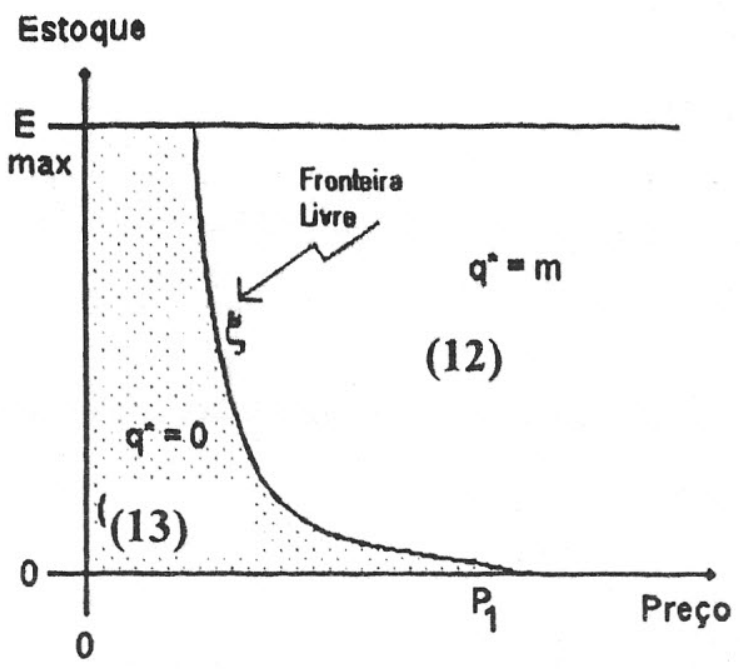

Figura 2 - Superfície livre no espaço \{ preço - estoque \}

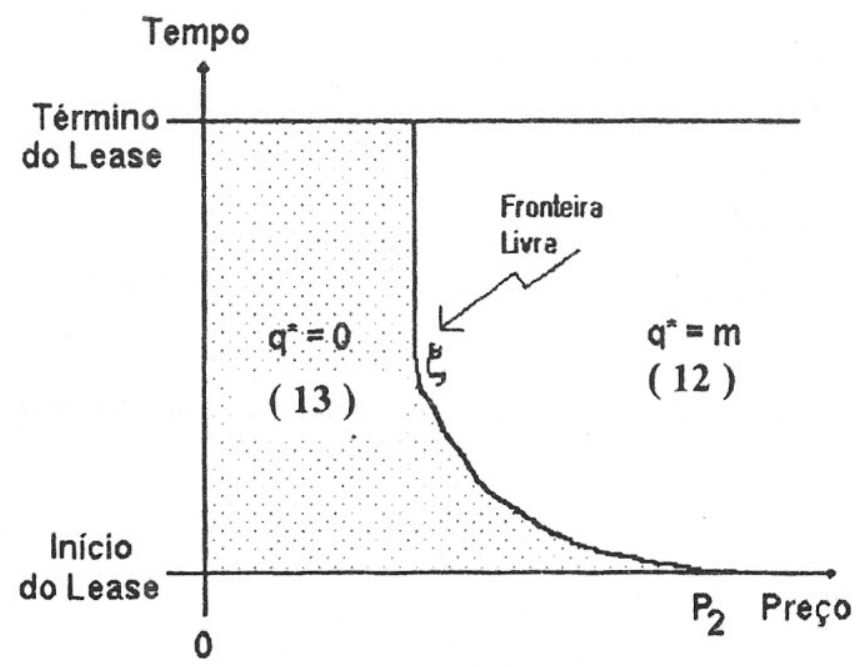

Figura 3- Superfície livre no espaço \{tempo, preço\}

Este é um problema de fronteira livre em duas dimensões, não se sabe a priori, antes de resolver o sistema formado pelas equações (12) e (13) e as suas condições de contorno, determinadas na seção 5.2, qual é exatamente a superfície $\xi$.

Como este sistema não possui solução analítica conhecida, a superfície $\xi$ somente pode ser determinada aproximadamente durante a solução numérica do problema. 
Substituindo (13) em (11):

$\left.\left(1-\tau_{c}\right)\left[\left(1-\tau_{r}\right) P^{*}-c_{0}-c_{1} q^{*}\right)\right]-(\lambda+r) V+V_{1}+(r-k) P V_{p}+\left[\mu E-q^{*}\right] V_{c}+1 / 2 \sigma_{p}{ }^{2} P^{2} V_{p p}+$ $+1 / 2 \sigma_{c}^{2} E^{2} V_{c c}=0$

A equação(14) vale no caso do projeto estar produzindo a taxa máxima, $q^{\bullet}=\beta$.

Substituindo (13) em (11) :

$V_{1}-(\lambda+r) V+(r-\kappa) P V_{p}+\mu E V_{c}+1 / 2 \sigma_{p}^{2} P^{2} V_{p p}+1 / 2 \sigma_{c}^{2} E^{2} V_{e c}=0$

A equação (15) vale no caso da exploração estar suspensa.

\section{2- Condiçð̃es de contorno}

As equações (14) e (15) estão sujeitas as seguintes condições de contorno:

- Condição terminal da concessão: no tempo final $T$, o valor da concessão é igual a zero:

$V(P, E, s)=0$, para $s=T$

- Se o preço da madeira chegar a zero, o processo estocástico (1), por ter uma barreira èm zero, fixa o valor da concessão em zero, mesmo que $\mathrm{t}<\mathrm{T}$ :

$\mathrm{V}(\theta, \mathrm{E}, \mathrm{s})=0, \mathrm{~s} \in\left[\mathrm{t}_{0}, \mathrm{~T}\right]$

- Existe uma densidade máxima de árvores na foresta, $E_{\max }$ Se o estoque atingir esta quantidade, será imposto que a derivada parcial do valor da concessão em relação ao estoque seja nula: 


$$
\frac{\partial(P, E, s)}{\partial E}=0 \text { no ponto } \mathbf{E}=\mathrm{E}_{\max } \quad \mathrm{s} \in\left[\mathrm{t}_{0}, \mathrm{~T}\right]
$$

- Existe um estoque mínimo de árvores na floresta $\mathrm{E}_{\min }$, se este nível mínimo for atingido a exploração deve parar:

$$
Q(P, E, s)=0, \forall s \in\left[t_{0}, T\right] \text {, se } \mathbf{E} \leq E_{\min }
$$

Para simplificar a resolução esta condição será substituida por:

$V\left(P, E_{\min }, t\right)=0$

ou seja, se o estoque atingir $\mathrm{E}_{\min }$, o direito de continuar explorando a floresta acaba.

- No limite, quando o preço tende para infinito, tenta-se explorar toda a reserva disponivel imediatamente. Neste caso o valor do projeto é função apenas do fluxo de caixa instantâneo advindo ao se exaurirem as reservas. Logo temos que:

$$
\begin{aligned}
& \mathrm{V}(\mathrm{P} \rightarrow \infty, \mathrm{E}, \mathrm{s})=\mathbf{F}\left(\mathrm{P}, \mathrm{E}, \pi ; \lambda ; \tau_{\mathrm{c}} ; \tau_{\mathrm{r}} ; \mathrm{E}_{\min }\right), \mathrm{s} \in\left[\mathrm{t}_{0}, \mathrm{~T}\right] \\
& \mathrm{V}=\left\{(1-\mathrm{tc})\left[(1-\mathrm{tr}) \times \mathbf{P} \times\left(\mathbf{E}-\mathrm{E}_{\min }\right)\right]-\pi\left(\mathrm{E}-\mathrm{E}_{\min }\right)\right\}-\lambda \mathbf{V} \\
& \mathrm{V}(1+\lambda)=\left\{(1-\mathrm{tc})\left[(1-\mathrm{tr}) \times \mathbf{P} \times\left(\mathbf{E}-\mathrm{E}_{\min }\right)\right]-\pi\left(\mathrm{E}-\mathrm{E}_{\min }\right)\right\}
\end{aligned}
$$

Ou seja:

$$
\lim _{P \rightarrow \infty} \frac{\partial V(P, I, s)}{\partial P}=\left(1-\tau_{c}\right)\left(1-\tau_{\mathrm{r}}\right)\left(\mathbf{E}-\mathrm{E}_{\min }\right) /(1+\lambda)
$$

A figura 4 representa as condições de contorno no espaço ( tempo, preço, estoque). 


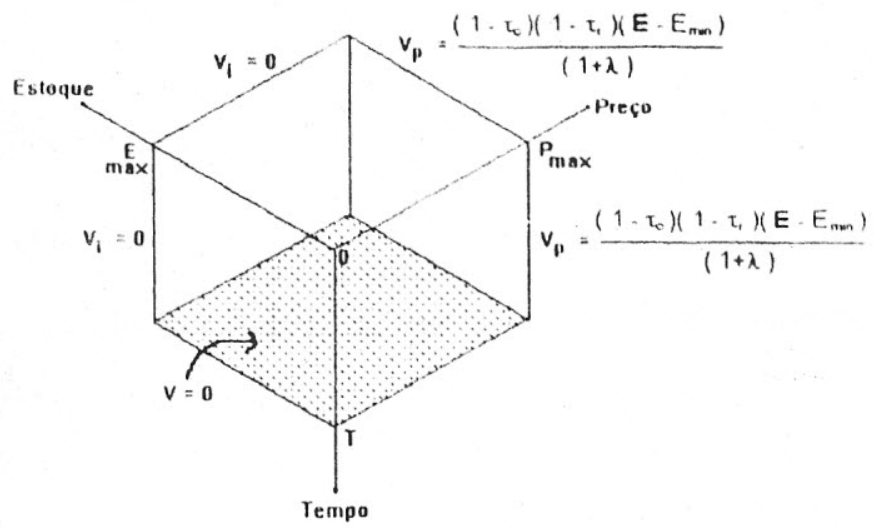

Figura 4-As condições de contorno no espaço \{tempo, preço, estoque

5.3- Aplicação à avaliação de uma concessão para a exploração de uma reserva florestal

Os dados abaixo, obtidos por Mork, Schwartz e Stangeland [17], provêem de um projeto tipico de exploração florestal na provincia de Alberta no Canadá:

Estoque Inicial de Madeira

Taxa média de crescimento

Desvio Padrão

Taxa de corte máxima

Maior Estoque possível

Preço inicial

Taxa média de crescimento

Desvio padrão

Imposto de Renda

Royalties

Imposto territorial

Custo Fixo

Custo Variável
$150.000 \mathrm{~m}^{3}$

$1,7 \%$ a.a.

$9,3 \%$ aa.

$17.753 \mathrm{~m}^{3}$ a.a.

$500.000 \mathrm{~m}^{3}$

$\$ 50 / \mathrm{m}^{3}$

$0,4 \%$ a.a.

$10,4 \%$ a.a.

$33 \%$ a.a.

$5 \%$ a.a.

$2 \%$ a.a.

$\$ 100.000$ a.a.

$\$ 33.8 / \mathrm{m}^{3}$

Custos Operacionais ( empregando a taxa de corte máxima ) $\$ 700.000$ a.a.

Duração da concessão

1C anos

Tabela 1 - Dados relativos ao projeto de exploração florestal 
Considera-se constante a taxa de crescimento do preço da madeira, e igual a sua média histórica. Também considera-se que a taxa de crescimento da floresta é constante e igual a sua média histórica. São utilizados dados históricos deflacionados para o desvio padrão do crescimento do preço e para o convenience yield da madeira.

\section{4 - Apresentação e análise dos resultados obtidos}

Foi desenvolvido um software em Microsoft ${ }^{\circledast}$ Qbasic $^{\circledast}$ e em Microsoft ${ }^{\circledast}$ Excel $^{\star}$, para rodar em microcomputadores tipo IBM-PC com microprocessador Pentium. O programa foi rodado no Laboratório de Computação do Departamento de Engenharia Industrial da PUC-Rio. Basicamente o software permite, utilizando o método explicíto de diferenças finitas, o cálculo de $\mathrm{V}(\mathrm{P}, \mathrm{E}, \mathrm{t})$ resolvendo numericamente a equação (14) $\mathrm{cm}$ conjunto com suas condições de contorno (16) a (20).

\begin{tabular}{|c|c|c|c|c|c|c|c|c|c|c|c|}
\hline 0 & 50 & 100 & 150 & 200 & 250 & 300 & 350 & 400 & 450 & $500^{\circ}$ & \\
\hline 0 & 0 & 0 & 0 & 0 & 0 & 0 & 0 & 0 & 0 & 0 & 00 \\
\hline 0 & 79 & 91 & 92 & 92 & 92 & 92 & 92 & 92 & 92 & 92 & 04 \\
\hline 0 & 131 & 170 & 178 & 179 & 179 & 179 & 179 & 179 & 179 & 179 & 08 \\
\hline 0 & 166 & 234 & 254 & 259 & 260 & 260 & 260 & 260 & 260 & 260 & 12 \\
\hline 0 & 189 & 284 & 320 & 331 & 334 & 335 & 335 & 335 & 335 & 335 & 16 \\
\hline 0 & 204 & 322 & 376 & 396 & 403 & 405 & 405 & 405 & 405 & 405 & 20 \\
\hline 0 & 214 & 351 & 423 & 453 & 465 & 469 & 470 & 470 & 470 & 470 & 24 \\
\hline 0 & 220 & 373 & 460 & 503 & 521 & 528 & 530 & 531 & 531 & 531 & 28 \\
\hline 0 & 225 & 389 & 491 & 545 & 570 & 581 & 586 & 587 & 588 & 588 & 32 \\
\hline 0 & 228 & 401 & 515 & 580 & 613 & 629 & 636 & 639 & 640 & 640 & 36 \\
\hline 0 & 230 & 410 & 535 & 610 & 651 & 672 & 682 & 687 & 689 & 690 & 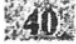 \\
\hline
\end{tabular}

Tabela 2 - Valor da concessão ( em milhares de dólares ), $P 0=50 \$ / \mathrm{m}^{3}$

Os resultados podem ser observados na tabela 2. A primeira linha da tabela representa o nível de estoque corrente em milhares de metros cúbicos, a última coluna representa o tempo remanescente em trimestres para o término dos direitos de explorar a reserva. A segunda linha indica a condição terminal da concessão, independente das dinâmicas do preço e do estoque. Decorridos 40 trimestres a concessão não vale mais nada. A primeira coluna indica que independente do tempo restante para o término dos 
direitos de exploração, se o estoque chegar a zero, a concessão também nào vale mais nada. Na interseção da quarta coluna com a última linha, esta indicado o equivalente ao "valor presente ". No início, existindo estoque de $150.000 \mathrm{~m}^{3}$ e estando o preço em $50 \$ / \mathrm{m}^{3}$, a concessão vale $\$ 535.000$.

A análise dos resultados deste problema que possui 3 variáveis, 17 parâmetros, e várias hipóteses simplificadoras, deve ser cuidadosa para não incorrer em erros de avaliação. Por exemplo, na tabela 2, a escala do estoque vai de zero a $500.000 \mathrm{~m}^{3}$. Os valores próximos a $500.000 \mathrm{~m}^{3}$ representam a situação limite descrita na condição de contorno (19). Perto desta situação limite, a precisão da aproximação obtida para a solução da EDP é bem menor. Portanto. deve-se eliminar as colunas correspondentes a um estoque inicial superior a $350.000 \mathrm{~m}^{3}$ para permanecer dentro da região em que a solução numérica é mais precisa. Também foi especificado na tabela 1 que o estoque inicial é de $150.000 \mathrm{~m}^{3}$ e a taxa de corte máxima é de $17.753 \mathrm{~m}^{3}$ por ano. Isto significa que em dez anos a exploração desta floresta pode render no máximo $177.530 \mathrm{~m}^{3}$ de madeira.

E importante frisar que os resultados obtidos e apresentados na tabela 2, correspondentes as colunas de estoque inicial superior a $150.000 \mathrm{~m}^{3}$, estão levando em conta que não será possivel cortar todas as árvores no tempo disponível. Este fato é devido que derivada parcial do valor da concessão em relação ao estoque ser praticamente nula para valores de estoque maiores que $150.000 \mathrm{~m}^{3}$.

Estoque $^{*} 1000 \mathrm{~m}^{3} \rightarrow$

\begin{tabular}{|rrrrrrrrr|}
\hline $\mathbf{0}$ & $\mathbf{5 0}$ & $\mathbf{1 0 0}$ & $\mathbf{1 5 0}$ & $\mathbf{2 0 0}$ & $\mathbf{2 5 0}$ & $\mathbf{3 0 0}$ & $\mathbf{3 5 0}$ & Custos Operacionais $\downarrow$ \\
\hline \hline 0 & 622 & 1110 & 1447 & 1651 & 1763 & 1819 & 1846 & $\mathbf{5 0 0 . 0 0 0}$ S/ano \\
0 & 462 & 824 & 1024 & 1225 & 1308 & 1350 & 1370 & $\mathbf{6 0 0 . 0 0 0}$ S/ano \\
0 & 301 & 537 & 700 & 799 & 853 & 880 & 893 & $\mathbf{7 0 0 . 0 0 0 ~ \$ / a n o ~}$ \\
0 & 140 & 251 & 327 & 373 & 398 & 411 & 417 & $\mathbf{8 0 0 . 0 0 0}$ S/ano \\
\hline
\end{tabular}

Tabela 3- Sensibilidade do valor da concessão em relação ao custos operacionais 


\section{6- Avaliaçăo por meio das técnicas de simulaçåo - Valor Presente Estratégico}

Hillier e Lieberman [9] definem a simulaçåo como sendo o braço experimental da Pesquisa Operacional. A simulação normalmente envolve a construção de um modelo matemático que represente um sistema real. Se possivel construir um modelo matemático que seja uma idealização razoável do problema real e possua uma solução analítica, então não é necessário utilizar técnicas de simulaçåo. Entretanto, existem problemas complexos que nåo podem ser resolvidos analiticamente. Nestes casos, havendo disponibilidade de computadores de alto desempenho e baixo custo de CPU, a simulação é um caminho prático para a sua solução.

O método de simulação Monte Carlo utilizado neste trabalho tem como base a descrição formal de Hillier e Lieberman [9]. Esta metodologia também é empregada, entre outros, por Geske e Shastri [8] em conjunto com outros métodos numéricos num benchmark sobre vários métodos apropriados para o apreçamento de opções, e por Hull [10], no contexto do apreçamento de opções financeiras européias, que o classifica como útil e eficiente quando o payoff do ativo derivativo depende diretamente da evolução de uma ou mais variáveis estocásticas base.

Através da simulação destas variáveis estocásticas base, descrevem-se, a partir de um instante inicial, no qual o preço da madeira, o estoque de madeira e o tempo para o término da concessão são conhecidos, possíveis caminhos para a evolução do preço e do estoque que possam vir a ocorrer no futuro. Entretanto a dinâmica dos preços deve ser ajustada. O seu drift deve ser diminuído de $\lambda \sigma_{p}$ onde $\lambda$ é o preço de mercado do risco e $\sigma_{p}$ é a sua volatilidade.

Hull [10] mostra que caso a variável em questão seja o preço de uma commodity negociada no mercado, como é suposto acontecer com a madeira neste trabalho, o efeito deste ajuste é igualar o drift à taxa livre de risco menos o retorno de conveniência ( convenience yield).

- Equação que modela a incerteza econômica: 


$$
d P / P=\left(r_{f}-k\right) d t+\sigma_{p} d z_{p}
$$

A dinâmica do estoque não sofre alteração porque a incerteza técnica é descorrelacionada com a economia.

- Equação que modela a incerteza técnica:

$$
d E=[\mu E-Q(P, E, t)] d t+\sigma E d z_{e}
$$

- Também é necessário impor a condição que força a existência de um estoque minimo de árvores na floresta, $E_{\min }$. Se este nivel minimo for atingido, a exploração deve parar:

$\mathrm{Q}(\mathrm{P}, \mathrm{E}, \mathrm{s})=0, \forall \mathrm{s} \in\left[\mathrm{t}_{0}, \mathrm{~T}\right]$, se $\mathbf{E} \leq \mathrm{E}_{\min }$

Formalmente, para proceder a efetuar a simulação Monte Carlo é preciso discretizar o sistema composto pelas equações (21), (22) e (23). O tempo de vida do ativo derivativo ( a concessão ) é dividido em $n$ subintervalos de tamanho $\Delta t$.

$$
\mathrm{t} \in\left\{\mathrm{t}_{0}, \mathrm{t}_{0}+\Delta \mathrm{t}, \mathrm{t}_{0}+2 \Delta \mathrm{t}, \ldots, \mathrm{t}_{0}+\mathrm{n} \Delta \mathrm{t}\right\}, \mathrm{t}_{0}+\mathrm{n} \Delta \mathrm{t}=\mathrm{T}
$$

- Equação que modela a incerteza técnica em tempo discreto:

$$
\left.\mathrm{E}+\Delta \mathrm{E}=\mathrm{E} * \operatorname{Exp}\left\{\left[\mu \mathrm{E}-\mathrm{Q}-1 / 2 \sigma_{\mathrm{c}}{ }^{2}\right] \Delta \mathrm{t}+\sigma_{\mathrm{e}} \varepsilon \sqrt{ }(\Delta \mathrm{t})\right]\right\}
$$

$\varepsilon \sim N(0,1)$, é uma amostra retirada de uma distribuição normal padrão.

- Equação que modela a incerteza econômica em tempo discreto:

$$
\left.\mathrm{P}+\Delta \mathrm{P}=\mathrm{P} * \operatorname{Exp}\left\{\left[\left(\mathrm{r}_{\mathrm{f}}-\kappa\right)-1 / 2 \sigma_{\mathrm{p}}^{2}\right] \Delta \mathrm{t}+\sigma_{\mathrm{p}} \varepsilon \sqrt{ }(\Delta \mathrm{t})\right]\right\}
$$

$\varepsilon \sim \mathbf{N}(0,1)$, é uma amostra retirada de uma distribuição normal padrão.

- As condições iniciais do sistema são conhecidas:

$P\left(t=t_{0}\right)=P_{0}$

$E\left(t=t_{0}\right)=E_{0}$ 
A cada rodada ( simulation run ) è possível o cálculo de $\mathrm{m}$ valores adiante $\Delta t$ temporalmente do início da simulação para o preço e para o estoque. Estes valores sâo uma amostra do conjunto de todos os possíveis valores que o preço e estoque poderiam assumir. Conhecidos estes valores e dada a taxa de corte, pode-se a seguir calcular o fluxo de caixa gerado por cada um desses possiveis caninhos.

- Equações que modelam o fluxo de caixa após a incidência dos impostos ${ }^{6}$ :

$\mathbf{F}(t+\Delta t)=\{(1-t c)[(1-t r) \times \mathbb{P}(t) \times Q(t)]-\pi(t)\} \quad \operatorname{se} Q(t)>0$

$\mathbf{F}(t+\Delta t)=0$ se $Q(t)=0$

Numa primeira abordagem é suposta uma atitude passiva na gestão do projeto, tal como ocorre nos métodos tradicionais. A medida que os eventos vão se revelando não é tomada nenhuma atitude no sentido de explorar os dois lados da incerteza. Assim, a taxa de corte de madeira é suposta constante e igual a taxa de extração máxima, desde o início da exploração até o término da concessão ou a exaustão das reservas. Logo, tem-se que:

$$
Q(t)=\beta
$$

A segunda abordagem visa calcular o valor esperado do VPL-E. Ou seja, são consideradas as opções de parada temporária na exploração do projeto. Pela diferença com o valor encontrado na primeira abordagem, estima-se o valor da flexibilidade operacional. Neste caso, a medida que os eventos vão se revelando, para cada estado da natureza, a gerência do projeto age determinando a taxa de corte de madeira visando maximizar o seu próximo fluxo de caixa. Logo, tem-se que:

$$
\begin{aligned}
& Q(t)=\beta \quad \text { para }\left(1-\tau_{c}\right)\left[\left(1-\tau_{r}\right) P(t)-\pi(t)\right] \geq 0 \\
& Q(t)=0 \text { caso contrário }
\end{aligned}
$$

Pode-se descrever a simulação como iniciando no tempo $t_{0}$ quando o estoque inicial $E_{0}$ e o preço inicial $P_{0}$ são conhecidos. É então determinada a taxa de corte $Q_{0}$ e o

\footnotetext{
6 Ignora-se o imposto proporcional ao valor da concessão que foi considerado anteriormente para simplificar o modelo.
} 
fluxo de caixa $F_{1}$ referentes a extração e a venda da madeira extraida no intervalo de tempo entre $t_{0}$ e $t_{0}+\Delta t$. É suposto que o fluxo de caixa $F_{1}$ é contabilizado no tempo $t_{0}+$ $\Delta \mathrm{t}$.

A seguir, são simulados $\mathrm{m}$ possiveis valores de preço, válidos para a madeira extraída entre $t_{0}+\Delta t$ e $t_{0}+2 \Delta t$ e também os $\mathrm{m}$ possiveis valores para $o$ estoque existente no tempo $t_{0}+\Delta t$. Cada par ordenado $\left\{P_{1, m}, E_{1, m}\right\}$ corresponde a um possível estado da natureza no tempo $t_{0}+\Delta t$.

Na primeira abordagem, sem considerar ação gerencial e, na segunda abordagem, existindo a possibilidade de exercer uma opção de suspender temporariamente a produção ${ }^{7}$ visando maximizar o fluxo de caixa seguinte, são então determinadas $\mathrm{m}$ taxas de corte, $Q_{1,1}, Q_{1,2}, \ldots, Q_{1, m}$ e os $m$ fluxos de caixa $F_{2,1}, F_{2,2}, \ldots, F_{2, m}$ referentes a venda da madeira extraida no intervalo de tempo entre $t_{0}+\Delta t e t_{0}+2 \Delta t$. Estes fluxos de caixa são contabilizados no tempo $t_{0}+2 \Delta t$.

O esquema mostrado na tabela 4 indica em tempo discreto possiveis caminhos simulados para as dinâmicas do preço e do estoque, e os possiveis fluxos de caixa esperados advindos da exploração da reserva florestal. A śsimulação prossegue até o tempo $T=t_{0}+n \Delta t$, quando os direitos de exploração acabam.

Através da simulação Monte Carlo, revelam-se as distribuiçōes de probabilidade dos fluxos de caixa futuros advindos da operaçâo de um projeto ou do exercicio de uma oportunidade de investimento. Estes possiveis fluxos de caixa podem incorporar ou não o exercício de opções operacionais. Calculando-se o valor esperado destes fluxos de caixa simulados e levando estes valores ao presente, é possível calcular o valor esperado do VPL. Ressalta-se que o valor esperado do VPL calculado é único, não é uma distribuição de probabilidade de VPL's.

Ao término da simulação, calcula-se o valor esperado de cada fluxo de caixa e a seguir é preciso levar estes valores ao tempo presente $\left(t_{0}\right)$. Isto pode ser realizado

${ }^{7}$ Existindo ação gerencial este té um sistema dinàmiço com feedback. 
utilizando a taxa de juros sem risco, porque considera-se por suposição uma economia neutra ao risco.

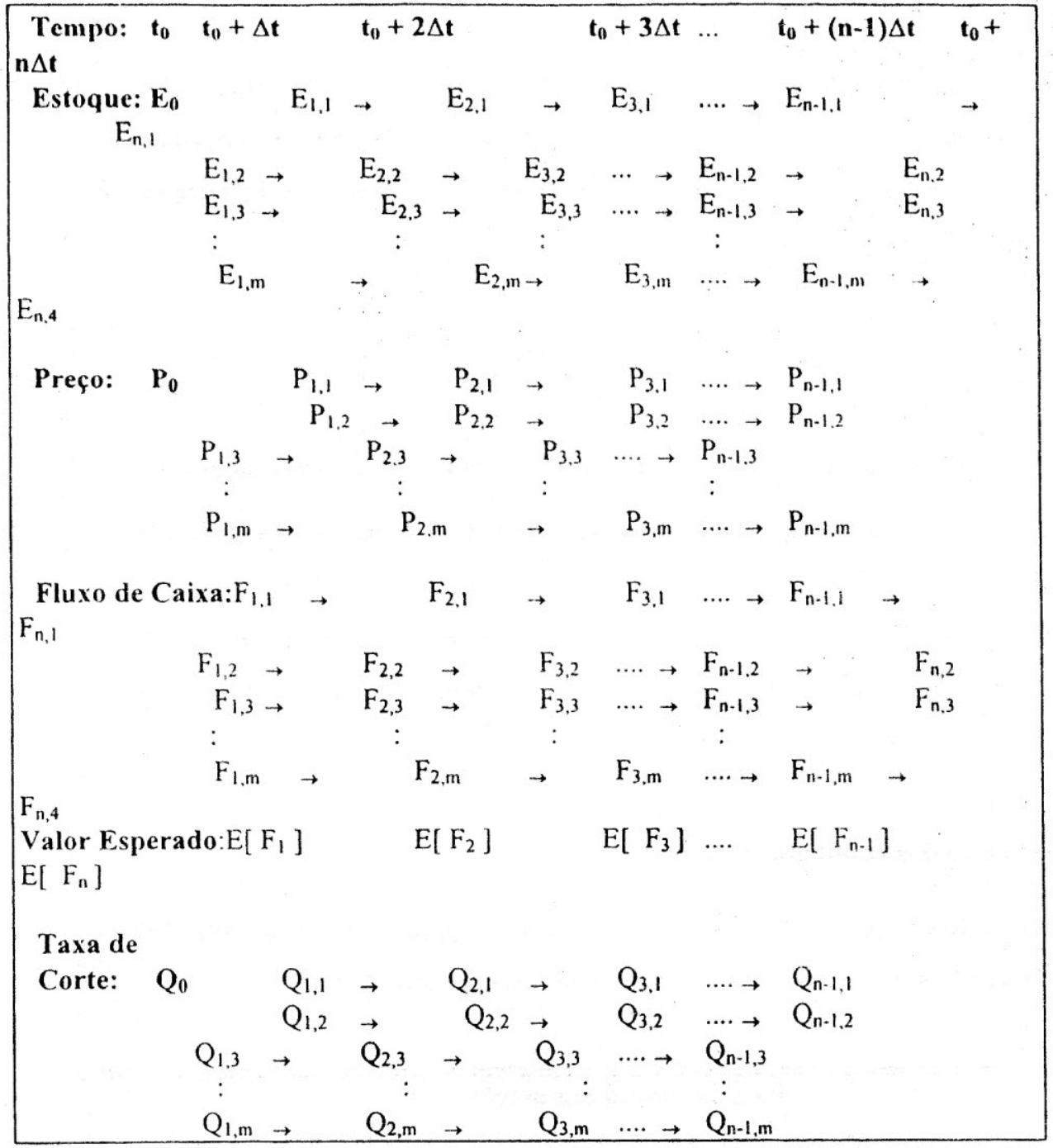

Tabela 4- Simulação Monte Carlo das dinâmicas do preço e do estoque Utilizando a primeira abordagem, que supõe a taxa de corte fixa, este valür corresponde a um VP-Dinâmico ${ }^{8}$, calculado em condições de incerteza:

\footnotetext{
${ }^{8}$ Neste problema VP e VPL se confundem porque năo existe investimento inicial.
} 


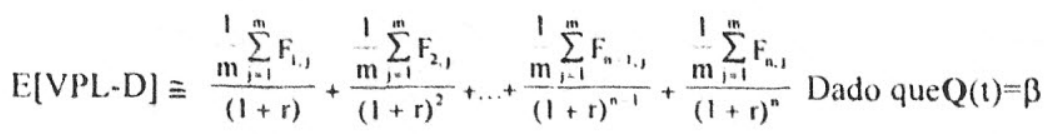

Utilizando a segunda abordagem, que supõe ação gerencial para tirar partido dos dois lados da incerteza através da possibilidade de exercer opções de suspender temporariamente a exploração da reserva, este valor corresponde ao VPL-Estratégico que incorpora os prêmios destas opções:

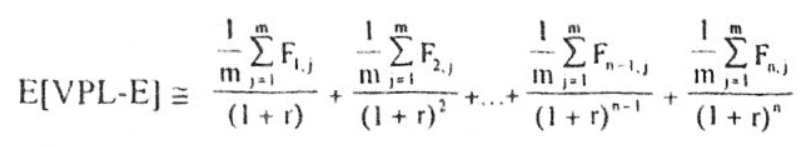

Dado que: $\quad \mathrm{Q}(\mathrm{t})=\beta$ para $\left(1-\tau_{\mathrm{c}}\right)\left[\left(1-\tau_{\mathrm{r}}\right) \mathrm{P}(\mathrm{t})-\pi(\mathrm{t})\right] \geq \mathrm{Q}(\mathrm{t})=0$ caso contrário

Finalmente, por diferença entre estes valores estima-se o valor da flexibilidade operacional. O valor da flexibilidade operacional é exacerbado neste modelo porque, por construção, não existem custos associados ao exercício da opção de suspender temporariamente a produção, e durante os períodos em que a extração é suspensa a firma também não incorre nos custos fixos.

\section{1- Apresentação dos resultados obtidos}

O algoritmo utilizado para a simulação Monte Carlo foi programado numa planilha Microsoft ${ }^{\circledR}$ Excel $^{\circledR}$. A entrada de dados desta planilha esta indicada na tabela 5 .

\begin{tabular}{|c|c|c|c|}
\hline \multicolumn{4}{|c|}{ Parâmetros da Simulação } \\
\hline Preço inicial $\left(\$ / \mathrm{m}^{3}\right)$ & 45 & Corporate tax rate ( $\%$ aa. ) & $33 \%$ \\
\hline drift preço ( $\%$ ao ano ) & $0,40 \%$ & Royalty tax rate ( $\%$ aa. ) & $5 \%$ \\
\hline desvio padrão preço ( $\%$ ao ano ) & $10,40 \%$ & Property tax rate ( $\%$ aa. ) & $0 \%$ \\
\hline convenience yield ( $\%$ ao ano ) & $4,60 \%$ & Custo Fixo (\$/ano) & 100.000 \\
\hline Estoque minimo $\left(\mathrm{m}^{3}\right)$ & 10.000 & Custo Variável $\left(\$ / \mathrm{m}^{3}\right)$ & 33.8 \\
\hline Estoque inicial $\left(\mathrm{m}^{3}\right)$ & 150.000 & $\beta$ & 17.753 \\
\hline drift anual estoque ( $\%$ ao ano ) & $1,70 \%$ & $\Delta \mathrm{t}($ anos $)$ & 1 \\
\hline desvio padrão estoque ( $\%$ ao ano ) & $9,30 \%$ & Risk free rate ( $\%$ aa. ) & $5,00 \%$ \\
\hline
\end{tabular}


Os resultados obtidos com os dados indicados na tabela 5, para o valor da concessão, são os seguintes:

\begin{tabular}{|l|l|}
\hline VP - D ( sem ação gerencial ): & $\$ 161.947$ \\
\hline VP - E ( com ação gerencial ): & $\$ 317.415$ \\
\hline $\begin{array}{l}\text { Estimativa do valor da } \\
\text { Flexibilidade Operacional: }\end{array}$ & $\$ 155.468$ \\
\hline
\end{tabular}

A figura 6 permite observar a assimetria que a existência de flexibilidade gerencial, modelada pela possibilidade de exercer opções de suspender temporariamente a produção, causa nas distribuições de probabilidade dos fluxos de caixa recebidos durante a exploração florestal.

Podemos observar na Figura 7 que o VPL-Dinâmico diminue na medida que o risco(volatilidade) associado ao projeto aumenta. Por outro lado, devido à existência da opção de poder suspender temporariamente a exploração da reserva, o VPL-Estratégico, que incorpora os prêmios desta opção, cresce na medida que o risco(volatilidade) aumenta. 


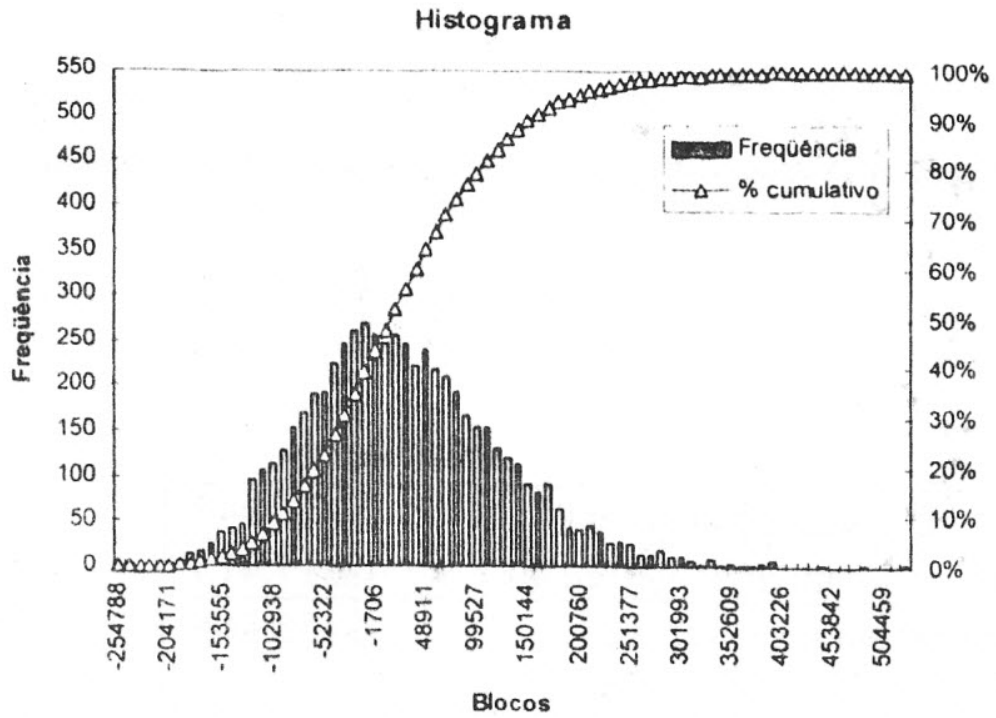

Figura 5 - Fluxos de caixa simulados sem ação gerencial

\section{Histograma}

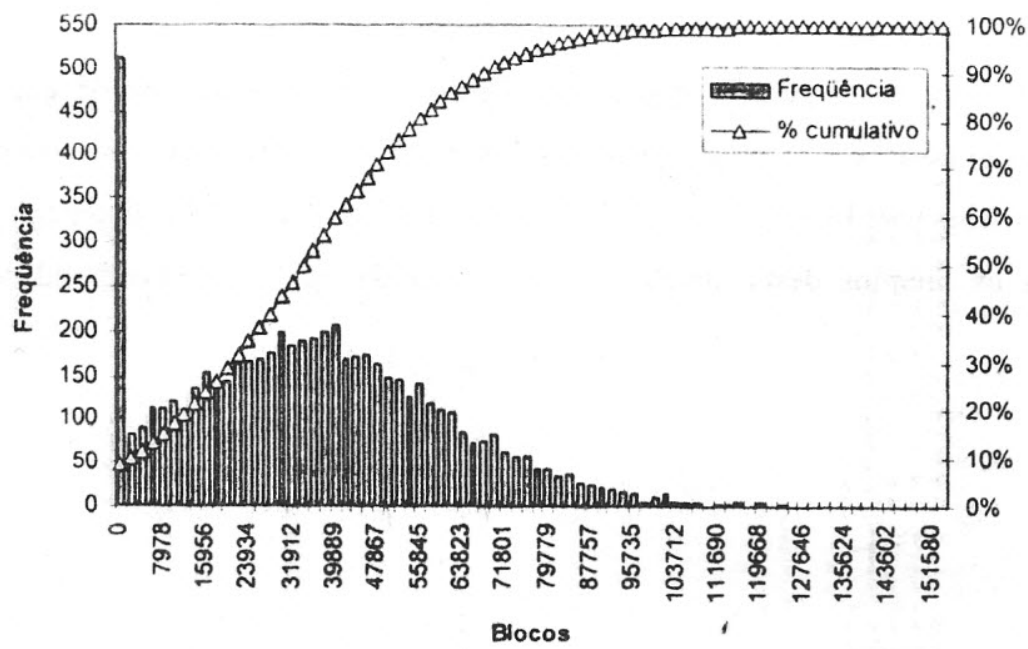

Figura 6 - Fluxos de caixa simulados considerando ação gerencial 


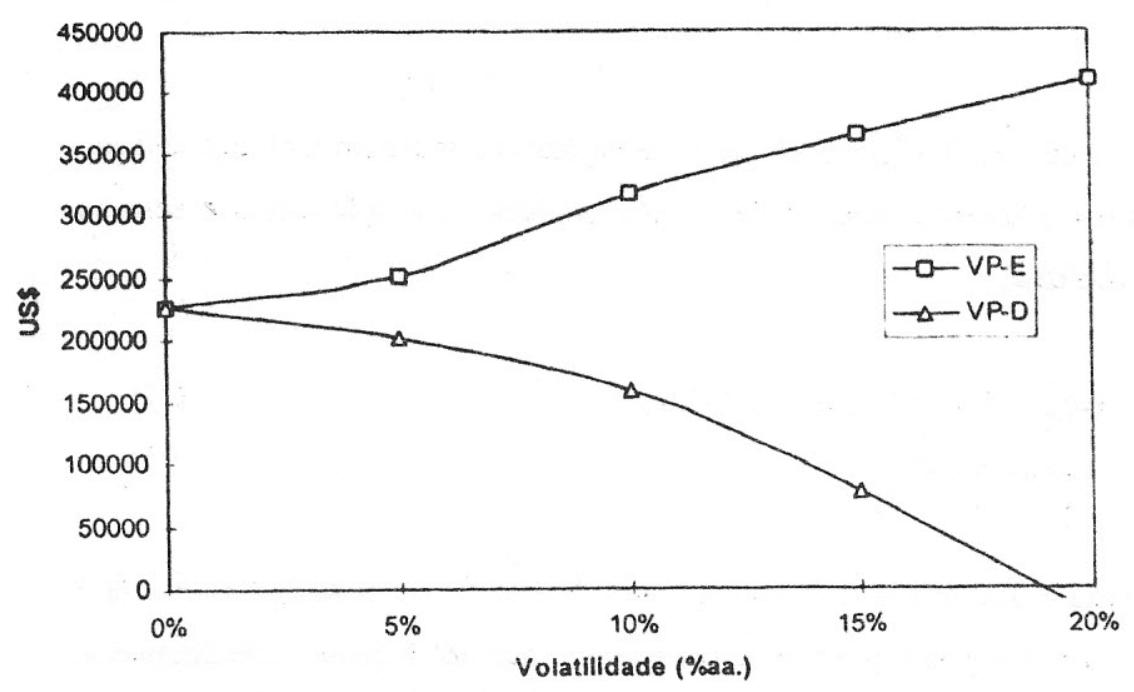

Figura 7 - Sensibilidade em relação a volatilidade, $P 0=\$ 45 / \mathrm{m} 3$

\section{7- Conclusões}

A metodologia desenvolvida neste trabalho para avaliação de concessões se diferencia do modelo apresentado por Mork, Schwartz e Stangeland [17] nos seguintes pontos:

- Sua derivação através da montagem de um portfolio livre de risco utilizando análise de ativos contingentes, não apresentou necessidade, como no modelo de MSS, de recorrer ao ICAPM(Intertemporal Capital asset Pricing Model). Porém foi preciso supor neutralidade ao risco oriundo da incerteza técnica. Entretanto, recorrendo ao ICAPM é possível resolver este problema considerando aversão ao risco. A análise econômica de projetos de exploração de petróleo sob condições de incerteza técnica e econômica foi assim resolvido por Aiube [1].

- A função de custo é suposta linear em relação à taxa de corte, ao invés de utilizar uma fuñção de custo quadrática, $\pi(Q)=c_{0}+c_{1} Q+c_{2} Q^{2}$ como no modelo de MSS. Esta simplificação procede porque a taxa de extração máxima é limitada tecnicamente, e 
dentro desta possivel faixa de variação da taxa de corte os custos săo aproximadamente lineares.

Se $\pi(Q)$ é linear em $Q$, o problema de controle ótimo estocástico terá uma soluçăo do tipo "produzir a máxima capacidade ou nåo produzir ", simplificando a soluçåo numerica do problema.

$$
\begin{array}{ll}
q^{\circ}=\beta & \text { para }\left(1-\tau_{\mathrm{e}}\right)\left[\left(1-\tau_{\mathrm{r}}\right) \mathrm{P}-\mathrm{c}_{1}\right]-\mathrm{V}_{\mathrm{e}} \geq 0 \\
\mathrm{q}^{\circ}=0 & \text { caso contrário }
\end{array}
$$

Mork, Schwartz e Stangeland [17] utilizam uma função de custo $\pi(Q)=c_{0}+c_{1} Q+$ $c_{2} Q^{2}$ e determinam a seguinte politica de corte ótima, que não assume necessariamente apenas os valores 0 ou maximo como a taxa de corte $q^{\bullet}$ calculada pelas equações (12) e (13).

Se houver não-linearidade dos custos com a taxa de corte, resulta uma EDP não linear relacionando o valor da concessão, ao preço, ao estoque e ao tempo. Pode-se fugir das complicações adicionais existentes na solução numérica de EDP's não lineares considerando uma função de custo linear por partes. Os resultados obtidos neste trabalho, comparados com o modelo não-linear, não diferem no pior caso por mais de $10 \%$.

Se for preciso considerar a não-linearidade, o método proposto neste artigo utilizando a simulação direta dos processos estocásticos base para calcular o valor esperado do VPL-E, é apropriado. É fácil de programar e dispensa a necessidade de conhecimentos avançados de análise numérica, necessários para a solução numérica de EDP's nãolineares.

\section{Referências Bibliográficas :}

[1] Aiube,F. Avaliação Económica de Projetos Petroliferos sob condições de incerteza de preços e reservas. Rio de Janeiro, Depto. Engenharia Industrial, PUCRJ, ( 1995) (Dissertação de Mestrado) 
[2] Black, F. \& Scholes, M. The pricing of options and corporate liabilities. Journal of political Economy \#81, (1973).

[3] Brennan,Michael J. e Schwartz,Eduardo S. Finite diference methods and jump processes in the pricing of contingent claims. Journal of Financial and Quantilative Analysis, September (1978)

[4] Brennan, M. J. The Price of Convenience and the Valuation of Commodity Contingent Claims. Stochastic Models and Options Value. New York, Noth Holland, (1991)

[5] Collatz,Lothar. The numerical treatment of differential equations. 2nd ed. SpringerVerlag, (1966)

[6] Dixit, A.K. \& Pindyck R.S. Investment under Uncertainty. Princeton University Press,(1994)

[7] Edelson,M. e Reinhardt F. Investment in Pollution Compliance Options:The Case of Georgia Power . Real Options in Capital Investments: models, strategies and aplications. Ed. by L. Trigeorgis, Praeger Publisher,(1995), pp.243-264.

[8] Geske,Robert e Shastri,Kuldeep. Valuation by aproximation: A comparision of alternative option valuation techniques. Journal of Financial and Quantitative Analysis, Vol. 20. March,(1985)

[9] Hillier,F.S. e Lieberman,G.J. Introduction to Operations Research. Holden-day,inc.

[10] Hull, J. C. Options, Futures, and Other Derivatives Securities. Prentice Hall, $2^{\text {a }}$ ed.(1993), Englewood Cliffs, NJ.

[11] Kulatilaka,N. The value of flexibility: A general model of Real Options. Real Options in Capital Investments: models, strategies and aplications. Ed. by L. Trigeorgis, Praeger Publisher, (1995), pp.89-108

[12] Laughton,D. e Jacoby,H. The effects of reversion on Commodity projects of different lenght. Real Options in Capital Investments: models, strategies and aplications. Ed. by L. Trigeorgis, Praeger Publisher, (1995), pp.185-206.

[13] Majd, S. e Pindyck, R.; Time to build, option value and investment decisions. Journal of Financial Economics, 18:7-27, March. (1987).

[14] Majd, S. e Pindyck, R.; "Appendix for time to build, option value and investment decisions" Journal of Financial Economics, 18:7-27, March. (1987).

[15] McDonald, R. \& D. Siegel. The Value of Waiting to Invest. Quarterly Journal of Economics, November (1986), pp.707-727.

[16] Merton, R. C. An Intertemporal Capital Asset Pricing Model. Econometrica \#41, (1973). 
[17] Mork R., Schwartz E. e Stangeland D. The valuation of forestry resources under stochastic prices and inventories. Journal of Financial and Quantitative Analysis, Vol.24, (1989).

[18] Pindyck, R.S. Irreversible Investment, Capacity Choice, and Value of the Firm. American Economic Review, 78: 969-985, December (1988).

[19] Pindyck, R.S. Investments of Uncertain Cost. Journal of Financial Economics, 34: 53-76, August. (1993).

[20] Samanez, C.P e De Oliveira, C. Avaliação e Gestão de Projetos de exploração de Recursos Naturais: Uma Abordagem pela Teoria das Opções. Revista Brasileira de Mercado de Capitais-RBMC, Vol.18:.63-76. (1993).

[21] Samanez, C.P e Batista, J. Influência da Incerteza, Postergação e Irreversibilidade dos Dispêndios em Decisões de Investimento. Revisıa Brasileira de Mercado de Capitais-RBMC, 20:7-22. (1995).

[22] Trigeorgis,L. The Nature of Options Interactions and the Valuation of Investments with Multiple Real Options. Journal of Financial and Quantitative Analysis, 28: 1-20, March (1993).

[23] Trigeorgis, L. A Log-Transformed Binomial Numerical Analysis for Complex Multi-Option Investments. Journal of Financial and Quantitative Analysis, Vol. 26 (1991)

[23] Tourinho, O. The valuation of reserves of natural resources: an option pricing approach. Berkeley, University of California, (1979). (PhD Thesis). 